4 Mangino D, Stover DE. Sarcoidosis presenting as metastatic bony disease. A case report and review of the literature on vertebral body sarcoidosis. Respiration 2004; 71: 292-294.

5 Talmi D, Smith S, Mulligan M. Central skeletal sarcoidosis mimicking metastatic disease. Skeletal Radiol 2008; 37: 757-761.

6 Binicer O, Sari I, Sen G, et al. Axial sarcoidosis mimicking radiographic sacroiliitis. Rheumatol Int 2009; 29: 343-345.

7 Aberg C, Ponzo F, Raphael B, et al. FDG positron emission tomography of bone involvement in sarcoidosis. AJR Am J Roentgenol 2004; 182: 975-977.
8 Mana J, Gomez-Vaquero C, Dorca J, et al. Vertebral and rib sarcoidosis: long-term clinical remission with methotrexate. Clin Rheumatol 1999; 18: 492-494.

9 Wilcox A, Bharadwaj P, Sharma OP. Bone sarcoidosis. Curr Opin Rheumatol 2000; 12: 321-330.

10 Zisman DA, Shorr AF, Lynch JP 3rd. Sarcoidosis involving the musculoskeletal system. Semin Respir Crit Care Med 2002; 23: 555-570.

\title{
Nodular pulmonary ossifications in differential diagnosis of solitary pulmonary nodules
}

\section{To the Editors:}

When considering a solitary pulmonary nodule or coin lesion, the differential diagnosis comprises a lot of different disease entities. Although uncommon, in specific patient groups, pulmonary ossifications should also be considered. We report two patients in whom the diagnosis of pulmonary ossification was confirmed pathologically and present a general discussion on this subject.

First, in a 68-yr-old patient with a history of hypercholesterolaemia and hypertension, a suspicious right mediastinal shadow was incidentally found on chest radiography after insertion of a central venous line. Personal history revealed poliomyelitis, retinal detachment and, especially, ventricular flutter for which he was resuscitated. Afterwards, he suffered from memory disturbances and even a frontal syndrome. A cardioverter-defibrillator was implanted. Chest radiography revealed a nodular aspect in the right lung hilum, which was not seen on a previous radiogram. Subsequent chest computed tomography (CT) demonstrated enlarged lymph nodes but no apparent ossifications. The mediastinum showed high metabolic activity on positron emission tomographic scan. Transbronchial biopsy was not conclusive. Cervical mediastinoscopy was negative. Subsequently, the right hilum was explored by thoracotomy. Enlarged lymph nodes were discovered and a wedge resection of the right middle lobe was performed to remove a calcified nodule of $\sim 1 \mathrm{~cm}$, surrounded by other small calcified lesions. Pathology demonstrated metaplastic bone tissue in the alveolar spaces and cores of ossification were diffusely spread across the pulmonary parenchyma. Diagnosis of diffuse nodular pulmonary ossification was made. Signs of pulmonary hypertension, such as intimal proliferation and focal myxoid degeneration of the parenchymal blood vessels were also present. Lymph nodes showed anthracosis and sarcoid reaction, but no signs of malignancy.

Secondly, a 71-yr-old patient with arterial hypertension and hypercholesterolaemia was diagnosed with a pT4N2M1 rectal carcinoma with liver metastases, for which he was treated by induction chemoradiation followed by rectal resection. After chemotherapy, a complete response of the liver metastases was initially achieved, but recurrent disease was diagnosed 1 yr later.
A wedge resection of two liver segments (II and VII) with cholecystectomy were performed followed by insertion of an intrahepatic PORT-A-CATH ${ }_{\circledast}$ system (Smiths Medical, Zaventem, Belgium). Pulmonary emboli occurred as postoperative complications. 6 months later, two other liver segments were resected (V and VI) and a further 3 months later, a right hepatectomy needed to be performed.

After diagnosis of two nodules in the right upper lobe suspicious for lung metastases 6 months after the hepatectomy, the patient was included in a phase II clinical trial investigating the effect of peroperative isolated lung perfusion with high-dose melphalan on lung metastases. The procedure was performed by an anterolateral muscle-sparing thoracotomy and, after lung perfusion, wedge excision of the suspicious nodules was performed. Pathology showed a metastasis of the colorectal carcinoma in one resection specimen and cores of ossification in the other, compatible with diffuse nodular pulmonary ossification. Remarkably, bone marrow was found in these ossification cores (fig. 1). All lymph nodes dissected during the procedure showed no signs of malignancy.

No post-operative complications were noted. Follow-up after 3 months revealed an uneventful course, with some tenderness remaining at the incision site.

Pulmonary ossification (PO) is an unusual condition where bone formation occurs within the parenchyma of the lung. Mostly, it is only recognised post mortem [1]. Ante mortem, it may be diagnosed radiographically [2] or after lung tumour resection, which is the case in our two patients. These POs can be spread diffusely in the lung, and may be divided into a nodular PO (NPO) and a dendritic PO (DPO), depending on their appearance on CT. Differentiation between DPO and NPO is presented in table 1 [1].

DPOs represent the less common form and are associated with pulmonary inflammation, pulmonary fibrosis, chronic obstructive pulmonary disease, asbestosis and pneumonia or may be idiopathic [3]. In a total of 1,393 adult autopsy cases performed by LARA et al. [1], only eight were identified as having dendritic calcifications, an average of $0.6 \%$ of all adult autopsies or one case every 3 yrs. The mechanisms behind the pathogenesis of $\mathrm{PO}$ still need further investigation. 


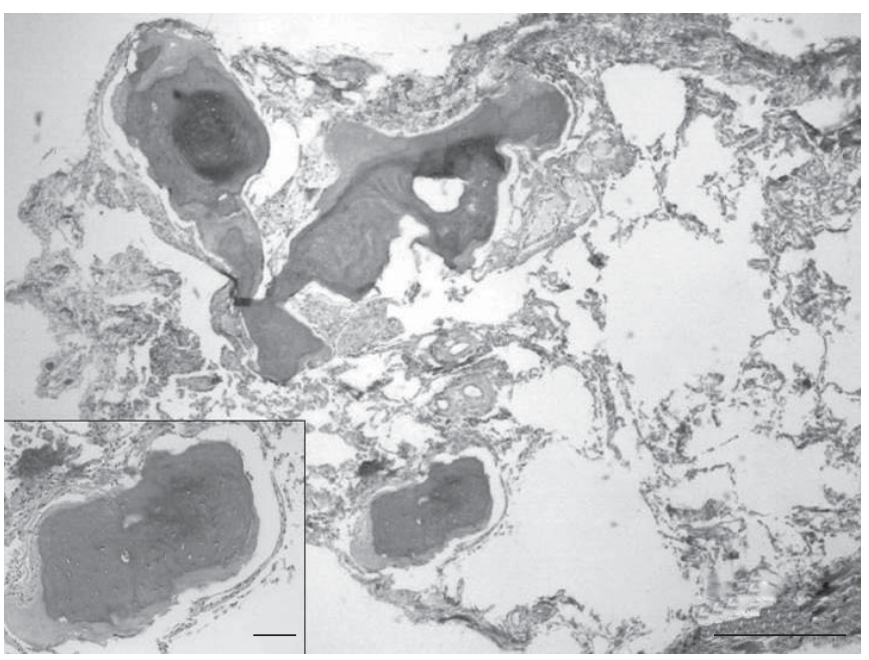

FIGURE 1. Diffuse nodular pulmonary ossifications. Multiple nodules of woven bone are present within the alveolar spaces. Scale bar $=500 \mu \mathrm{m}$. The inset shows a detail of the woven bone within an air space. Scale bar $=100 \mu \mathrm{m}$. Haematoxylin and eosin stain.

However, it seems that inflammation after tissue injury may result in DPOs through interaction between calcium and fatty acids from degraded phospholipids of the cell membrane. Alkaline phosphatase activity is elevated on the surface of the alveolar type II cells, which increases osteoblastic activity. Pulmonary fibrosis is associated with increased alveolar activity of this enzyme [4]. Transforming growth factor (TGF)- $\beta$ is present in lung fibrosis and stimulates production of collagen type II, proteoglycans and fibronectin, leading to fibrosis. TGF- $\beta$ may also provoke osteoblastic proliferation, possibly due to its similarities to bone morphogenic protein [5].

The mature bone tissue branches out through the interstitium, occasionally piercing through the tissue into the alveolar spaces, giving it a characteristic tree-like appearance on chest radiography. The centre of the ossification often consists of bone marrow, with fat or haematopoetic elements.

NPOs are more common and have been associated with chronic heart failure and mitral stenosis, resulting in passive congestion [6]. They may be caused by extravasation of red blood cells in passive congestion due to chronic heart failure. Lysis of the erythrocytes results in haemosiderin deposits [7] on the alveolar walls and inflammation, fibrosis and hyalinisation. These haemosiderin accumulations attract calcium salts and giant cells giving rise to "endogenous pneumoconiosis". The lesions are most commonly seen in the alveolar spaces of the inferior pulmonary lobe as a smooth rounded mass expanding the alveolus. Marrow formation is not as usual as in dendritic ossifications.

There is a great overlap between the two forms and both may occur at the same time in the same patient. Further investigation of the specimen reveals its dendritic or nodular nature, for instance when bone marrow is present. There is also a great overlap in pre-existing pulmonary and cardiac disorders in patients with pulmonary ossifications.

A relationship between diffuse pulmonary ossifications and diabetes mellitus has also been suggested, as it is often associated with diffuse pulmonary ossification. Diabetes results in elevated calcium deposition not only in atherosclerotic vessels but also throughout the entire body, so-called medial calcifications, which contain amorphous bone tissue and osteoclasts [8]. A possible pathogenic mechanism is the extension of nonatherosclerotic vascular calcification in the small pulmonary vessels towards the perivascular lung tissue.

Although uncommon, pulmonary calcifications require specific attention and awareness of the clinician, as they may be mistaken for other clinical disorders. They should be considered in the differential diagnosis of solitary pulmonary nodules, both clinically (peroperatively) and radiographically [9].

POs are regularly misinterpreted on CT examination. They can be misdiagnosed as bronchiectases, the ossification being mistaken for ectatic bronchioles, hamartomas with bone formation, or silicotic nodules. Further investigation with high-resolution CT generating bone window images may reveal the bony nature of the process. A characteristic popcorn calcification may be an indication of the chondroid calcification in a hamartoma, while eccentric calcification may represent a calcified granuloma surrounded by malignancy [10]. Silicotic nodules calcify rarely and are infrequently associated with thickened interlobular septa, which can be observed in sarcoidosis. Therefore, POs often go unrecognised and may result in overtreatment. Certainly, in elderly patients suffering from chronic lung disease or congestive heart failure, they should be included in the differential diagnosis of solitary pulmonary nodules.

\section{J. Gielis*, M. Torfs*, M. Luijks", R. Salgado and P.E. Van Schil* \\ *Dept of Thoracic and Vascular Surgery, "Dept of Pathology, and 'Dept of Radiology, Antwerp, University Hospital, Antwerp, Belgium.}

TABLE 1 Differentiation between dendritic (DPO) and nodular (NPO) pulmonary ossifications

Feature DPO
NPO
Round with smooth contours, lobulated Within alveolar spaces, lobulated bone Absent
Histological

Marrow

Clinic
Branching, dendritic; coral-like

In alveolar septa, tubular shape of bone

Present

Associated with chronic obstructive lung disease and interstitial fibrosis 
Correspondence: P.E. Van Schil, Dept of Thoracic and Vascular Surgery, Antwerp University Hospital, Wilrijkstraat 10, B-2650 Edegem, Antwerp, Belgium. E-mail: paul.van.schil@uza.be

Statement of Interest: None declared.

\section{REFERENCES}

1 Lara JF, Catroppo JF, Kim DU, et al. Dendriform pulmonary ossification, a form of diffuse pulmonary ossification: report of a 26year autopsy experience. Arch Pathol Lab Med 2005; 129: 348-353.

2 Jaderborg JM, Dunton RF. Rare clinical diagnosis of dendriform pulmonary ossification. Ann Thorac Surg 2001; 71: 2009-2011.

3 Felson B, Schwarz J, Lukin RR, et al. Idiopathic pulmonary ossification. Radiology 1984; 153: 303-310.

4 Capelli A, Lusuardi M, Cerutti CG, et al. Lung alkaline phosphatase as a marker of fibrosis in chronic interstitial disorders. Am J Respir Crit Care Med 1997; 155: 249-253.
5 Chan ED, Morales DV, Welsh CH, et al. Calcium deposition with or without bone formation in the lung. Am J Respir Crit Care Med 2002; 165: 1654-1669.

6 Joines RW, Roggli VL. Dendriform pulmonary ossification. Report of two cases with unique findings. Am J Clin Pathol 1989; 91: 398-402.

7 Colby TV. Miscellaneous conditions and lung diseases of unknown origin. In: Hasleton PS, ed. Spencer's Pathology of the Lung. New York, McGraw-Hill, 2009; pp. 792-795.

8 Abedin M, Tintut Y, Demer LL. Vascular calcification: mechanisms and clinical ramifications. Arterioscler Thromb Vasc Biol 2004; 24: 1161-1170.

9 Erasmus JJ, Connolly JE, McAdams HP, et al. Solitary pulmonary nodules: part I. Morphologic evaluation for differentiation of benign and malignant lesions. Radiographics 2000; 20: 43-58.

10 Leef JL III, Klein JS. The solitary pulmonary nodule. Radiol Clin North Am 2002; 40: 123-143.

\section{An autopsy case of pulmonary veno-occlusive disease refractory to imatinib}

\section{To the Editors:}

Pulmonary veno-occlusive disease (PVOD) is a rare variant of pulmonary hypertension (PH). PVOD is reportedly refractory to the majority of $\mathrm{PH}$-specific vasodilators; however, a recent case report demonstrated that a tyrosine kinase inhibitor, imatinib, markedly improved functional capacity in a patient with PVOD [1]. The present case report documents clinical and haemodynamic effects of imatinib in a patient with pathologically confirmed PVOD.

In 2007, a 73-yr-old female experienced progressive exertional dyspnoea and was diagnosed with $\mathrm{PH}$ in a community hospital. Diuretics and beraprost (oral prostanoid) were administered to no avail. The patient was referred to Hokkaido University Hospital (Sapporo, Japan) in November 2008. She had never smoked and had no occupational or environmental inhalation history.

Functional capacity was New York Heart Association class III. Blood pressure was 110/65 mmHg, and coarse crackles were present in both lungs. Serum aspartate aminotransferase and alanine aminotransferase levels were within normal range, whereas hepatitis B (HB) surface and HBe antigens were both positive. Serum brain-type natriuretic polypeptide was elevated at 2,456 $\mathrm{pg} \cdot \mathrm{mL}^{-1}$, and arterial blood gas analysis showed hypoxaemia (arterial oxygen tension $55.2 \mathrm{mmHg}$ ) and hypocapnia (arterial carbon dioxide tension $33.9 \mathrm{mmHg}$ ). Chest radiography demonstrated marked cardiomegaly, dilated main pulmonary artery and pulmonary congestion. Echocardiography showed marked right atrial and ventricular enlargement and severe tricuspid regurgitation. Pulmonary function tests revealed normal forced vital capacity (FVC), forced expiratory volume in $1 \mathrm{~s}$ (FEV1) and FEV1/FVC, whereas diffusing capacity of the lung for carbon monoxide
(DL,CO) and DL,CO/alveolar volume (VA) were markedly reduced: $D \mathrm{~L}, \mathrm{CO} 4.16 \mathrm{~mL} \cdot \mathrm{min}^{-1} \cdot \mathrm{mmHg}^{-1}, 33.5 \%$ of predicted; $D \mathrm{~L}, \mathrm{CO} / V \mathrm{~A} 1.61 \mathrm{~mL} \cdot \mathrm{min}^{-1} \cdot \mathrm{mmHg}^{-1} \cdot \mathrm{L}^{-1}, 37.4 \%$ pred. High-resolution computed tomography (HRCT) revealed slight but diffuse ground-glass opacities, thickening of the septal lines, and hilar and mediastinal lymphadenopathy (fig. 1). Right heart catheterisation performed at this time exhibited elevated mean pulmonary artery pressure $\left(\bar{P}_{\mathrm{pa}}\right)$ and pulmonary vascular resistance (PVR) along with slightly reduced cardiac output (CO): $\bar{P}_{\text {pa }} 53 \mathrm{mmHg}$, pulmonary capillary wedge pressure $10 \mathrm{mmHg}, \mathrm{CO} 3.45 \mathrm{~L} \cdot \mathrm{min}^{-1}$ (cardiac index $2.63 \mathrm{~L}$. $\mathrm{min}^{-1} \cdot \mathrm{m}^{-2}$ ), right ventricular end diastolic pressure $14 \mathrm{mmHg}$, right atrial pressure $7 \mathrm{mmHg}$, and PVR 1,159 dyn $\cdot \mathrm{s} \cdot \mathrm{cm}^{-5}$.

With a clinical diagnosis of PVOD, an oral phosphodiesterase-5 inhibitor, sildenafil, was started in December 2008 but hypoxia and pulmonary haemodynamics deteriorated. The endothelin receptor antagonist bosentan was not initiated because of comorbid HBe antigen-positive HB. The intravenous prostacyclin epoprostenol was not used because we feared a lifethreatening deterioration of pulmonary oedema and hypoxaemia, although a recent report suggested that it can have a beneficial effect on PVOD when used with caution [2]. Lung transplantation is the optimal treatment for PVOD, and we considered the patient's suitability for this procedure; however, we concluded that the patient would not be a candidate because of her age, poor functional status, estimated long waiting time and the possibility of relapse of multiple myeloma. After reading a recent case report in which a patient with typical features of PVOD responded well to imatinib [1], we decided to use this agent in our patient. We administered imatinib (Gleevec ${ }^{\circledR}$; Novartis Pharmaceuticals, Basle, Switzerland) in February 2009 after obtaining approval from the ethical committee of our institution and written informed 